\title{
"La tierra clama por remedio": la conquista espiritual del territorio muisca
}

\author{
J. Michael Francis \\ University of North Florida
}

\begin{abstract}
Resumen
La historiografía colonial de América Latina ha enfatizado el papel central y decisivo que jugó la Iglesia Católica en el desarrollo de la conquista, colonización y transformación cultural de los habitantes del Nuevo Mundo. Pero la verdad es que sabemos muy poco sobre el desarrollo del cristianismo en los inicios de la colonización neogranadina. La mayoría de los estudios coloniales sobre territorio muisca no considera el papel de la Iglesia. Este estudio, enfatizando los obstáculos que enfrentaron los clérigos y los esfuerzos hechos para convertir a la población indígena, pone en duda el concepto popular de que la conquista espiritual de los muiscas fue rápida y completa. Este artículo, fruto de un cuidadoso análisis en los archivos coloniales de Colombia, España e Italia, debe ser considerado como un primer paso en la difícil reconstrucción de un proceso prolongado y muy complejo.
\end{abstract}

\begin{abstract}
The historiography of colonial Latín America has emphasized the central and decisive role of the Catholic Church in the conquest, colonization and cultural transformation of the inhabitants of the New World. This paper is an initial step toward understanding the role of the Church in the evangelization of the Muisca Indians in early-colonial New Granada, a topic that has received surprisingly little scholarly attention. A careful analysis of colonial records from Colombia, Spain and Italy suggests that the Church in early-colonial New Granada had limited influence in the daily lives of the Muisca inhabitants of Colombia's Eastern Highlands.
\end{abstract}




\section{FRONTERAS}

delahistoria

Vol 5 / 2000

\section{Introducción}

"i Vicente de Requejada ha muerto! Lo han matado. Aquellos indios miserables, desdeñosos de Garagoa se han rebelado contra nosotros y han asesinado al pobre Fray Vicente de Requejada". Tales noticias alarmaron a los habitantes de la recientemente fundada ciudad de Tunja, una población que para 1541 emergía como uno de los centros españoles más importantes de la Nueva Granada. El fundador de Tunja, Gonzalo Suárez Rondón, había presenciado el ataque al fraile agustino y testificó que había visto a varios indígenas de Garagoa llevando algunos jirones ensangrentados del hábito de Requejada. Como es de esperarse, mientras Suárez y sus hombres huían del pueblo sublevado y retornaban a la seguridad que Tunja les brindaba, asumieron que el fraile había muerto. Sin embargo, Requejada había sobrevivido al ataque. Algunos días después, el atemorizado y golpeado fraile apareció en Tunja, "completamente desnudo, menos la paja que cubría sus vergüenzas, y tan débil de hambre que casi no se podía parar"1 ${ }^{1}$. Más adelante Requejada explicó que había pasado la mayor parte de aquellos difíciles días escondido bajo rimeros de paja; sólo de noche, bajo el amparo de la oscuridad, se había atrevido a aventurarse y salir, esperando eludir a los indios que lo perseguían ${ }^{2}$.

Pero ¿qué fue lo que hizo el pobre fraile? ¿Por qué lo atacaron los indígenas de Garagoa? Infortunadamente las fuentes coloniales ofrecen pocas claves para explicar las circunstancias que rodearon el levantamiento. Los recuentos del incidente son tanto vagos como contradictorios ${ }^{3}$. Los testigos españoles aseguraron que no hicieron nada para generar el conflicto; a pesar de ello, los detalles del incidente que fueron narrados por los nativos de Garagoa contrastan fuertemente con los testimonios españoles. Ellos insisten en que fueron víctimas inocentes de un brutal

\footnotetext{
${ }^{1}$ Archivo General de Indias, Sevilla (en adelante citado como AGI), Justicia, 497, bl. 4, Testimonio de Francisco Rodríguez (1562).

${ }^{2}$ AGI, Justicia, 497, bl. 4 (1562).

${ }^{3}$ Los españoles que presenciaron el ataque afirman que fueron los indios de Garagoa los que iniciaron los enfrentamientos cuando, armados con lanzas y macanas de guerra, lanzaron un ataque sorpresivo sobre un pequeño grupo de españoles que simplemente pasaban por el sector. Según varios españoles que estaban presentes, el ataque no fue producto de una provocación; sin embargo, si ese fue el caso, el grupo de conquistadores, incluyendo a Requejada, corrió con gran suerte pues no sufrió ninguna baja (aunque un número indeterminado de sus aliados indígenas murió a manos de los guerreros de Garagoa). Ver Archivo General de la Nación, Bogotá (en adelante citado como AGN), Caciques e Indios, 35, n. 25, fols. 707-717.
} 
asalto a su pueblo, un asalto que no fue provocado y que empezó cuando los españoles intentaron saquear algunos recintos sagrados ${ }^{4}$. Además, agregaron los testigos, lo que siguió fue realmente una masacre: cuatrocientas personas de Garagoa, de acuerdo con los relatos de los nativos, perdieron sus vidas defendiendo al pueblo de los españoles y sus aliados indígenas.

A pesar de la naturaleza lacónica de los registros coloniales, hay indicios convincentes de que la presencia española ese día en Garagoa no fue del todo inocente. El conflicto coincidía con la expedición masiva de Hernán Pérez de Quesada, que iba en busca de la Casa del Sol. Se trataba de un santuario muisca que se había convertido en un verdadero El Dorado a los ojos de los españoles, ávidos de encontrar su ubicación. Se rumoraba que era un recinto funerario para los señores muiscas, cuyas tumbas estaban llenas de inimaginables cantidades de oro, esmeraldas y otros objetos preciosos. Aunque nada sugería que la Casa del Sol estuviese cerca a Garagoa, para 1541 era saber popular que los cementerios muiscas contenían increíbles riquezas. De otro lado, Garagoa no fue el único pueblo que Hernán Pérez de Quesada y sus hombres saquearon. Como es de esperarse, los habitantes Muiscas del pueblo objetaron la profanación de sus santuarios y en respuesta montaron una feroz, aunque costosa, resistencia. Vicente de Requejada era uno de los trescientos españoles que se ofrecieron para la expedición de Hernán Pérez y probablemente fue su participación en el saqueo de los recintos de Garagoa lo que propició la ira de los indígenas y casi le cuesta la vida.

Pero, ¿qué tiene que ver la participación de Requejada en los eventos de Garagoa con sus responsabilidades y expectativas como fraile? ¿Qué puede decimos acerca de la conquista espiritual del territorio muisca? Sabemos muy poco sobre la vida de

\footnotetext{
${ }^{4}$ Para un breve recuento del ataque a Garagoa, ver AGN, Caciques e Indios, 35, n. 25, fols. 707-717 y AGI, Justicia, 497, bl. 4 (1562). También se ha de notar que el conflicto en Garagoa, aunque raro, no fue un incidente aislado a pesar de que los estudiosos a menudo describen la conquista del territorio muisca y su ransición al orden colonial como un proceso notablemente pacífico. Se podría enfatizar que un año después de su llegada a la Nueva Granada, sólo seis de los conquistadores originales perdieron sus vidas, ninguno a manos de un indígena. De otro lado, sí hubo algunas revueltas menores en Ceniza, Suta, Tausa, Cucunubá, Ocavita, Subachoque, Boyacá y Tinjacá. En Duitama y Sogamoso se presentaron levantamientos serios que fueron reprimidos de manera brutal (con pura sangre) por el alguacil mayor de Tunja, el capitán Baltasar Maldonado. Ver Fray Pedro de Aguado, Recopilación Historial, vol. 1, lib. 4, cap. 10 (Bogotá: Biblioteca de la Presidencia de Colombia, 1957), 297, 298.
} 


\section{FRONTERAS}

los miembros del clero en las etapas más tempranas de la Nueva Granada; carecemos para este territorio, de los valiosos estudios prosopográficos que han sido de gran utilidad para comprender la situación del clero colonial mejicano. Teniendo esto en cuenta, sería una irresponsabilidad académica y una pésima interpretación asumir simplemente que las acciones y experiencias de Vicente de Requejada reflejan toda la gama de experiencias que vivieron los primeros religiosos de la Nueva Granada. Después de todo, según lo que sabemos, Requejada era un individuo bastante peculiar. Fue uno de los primeros españoles en entrar a la Nueva Granada, sirviendo de capellán a la expedición de Nicolás de Federmán que provenía de Venezuela en $1535^{5}$. Antes de que se estableciera la Audiencia en 1550, poseía ilegalmente su propia encomienda. No sólo esto, sino que luego abandonó la orden agustina, para convertirse en un sacerdote seglar y un acaudalado terrateniente. Además, consolidó una unión ilícita con una indígena, con quien tuvo un hijo. En su testamento Requejada, arrepentido, reconoció sus faltas como sacerdote y dejó como herencia 100 pesos de oro a cada uno de los miembros indígenas de su parroquia en Coromoro. Dejó otros 100 pesos a los nativos de Foacá y 50 pesos a los habitantes de Samacá .

No cabe duda que Requejada se destaca como una figura única en la conquista espiritual de la Nueva Granada. Era un individuo excepcional cuyas acciones y experiencias contrastan ampliamente con las de otros frailes y sacerdotes que lucharon a favor de los nativos. Tales individuos existían y, en general, las relaciones entre el clero y la población indígena en el territorio muisca fueron pacíficas. Quizás la evidencia más convincente al respecto sea que aparte de Vicente de Requejada y su encuentro cercano con la muerte en Garagoa, y el incidente menor del franciscano Antonio de Alcántara en 1574 en el pueblo de Tota, la provincia colonial de Tunja nunca se acercó siquiera a producir su propio mártir ${ }^{7}$.

\footnotetext{
${ }^{5}$ Nacido en España entre 1498 y 1501, Requejada llegó al Nuevo Mundo en abril de 1529 como miembro de una expedición armada, desde la provincia de Venezuela. Ya que era uno de los primeros frailes de la Nueva Granada debió tener muy poca supervisión eclesiástica. Para mayor información sobre sus años en Venezuela, ver José Ignacio Avellaneda Navas, Los Compañeros de Federmán (Bogotá: Tercer Mundo, 1990), 292-297.

${ }^{6}$ Archivo Regional de Boyacá, Tunja, leg. 10, fol. 99r. Vicente de Requejada murió en 1574 o 1575, más de treinta años después de los eventos de Garagoa.

${ }^{7}$ Alcántara se opuso a que uno de los miembros de su parroquia saliera temprano de misa, y cuando el anciano indígena se negó a oírlo, el fraile lo tiró de los cabellos. Los indígenas de Tota se opusieron a sus acciones y en respuesta le lanzaron piedras. Una de ellas le rompió una costilla. Ver AGN, Caciques e Indios, 29, n. 1, fols. 123r-124r.
} 
Sin embargo, la poca frecuencia con que se presentaban confrontaciones físicas entre el clero y la población nativa puede llevar a conclusiones erróneas. Hay que reconocer que en el siglo XVI, el contacto diario entre los sacerdotes y los indígenas era extremadamente raro, $\mathrm{y}$ las fuentes apenas ofrecen vistazos breves de las interacciones que se llevaban a cabo. Lo cierto es que la información disponible pone en duda muchos de los supuestos que manejamos respecto al papel que cumplía la Iglesia a principios del periodo colonial en la Nueva Granada y en América Latina en general. Los registros coloniales siembran muchas dudas sobre la influencia del clero y del cristianismo en la vida cotidiana de la población indígena neogranadina. Más aún, nos obligan a examinar con más cuidado las vidas de quienes guiaron la campaña espiritual en el territorio muisca. Hay pocos indicios de la "pureza apostólica" que se les atribuía a los primeros religiosos de la Nueva España ${ }^{8}$. Por el contrario, las fuentes revelan a un clero motivado más por la codicia que por una genuina preocupación vinculada a la salvación de los indígenas. Desde luego, la complejidad de este tema no se debe subestimar. El clero de la Nueva Granada se enfrentó a abrumadores obstáculos; para empezar, eran pocos comparados con la población indígena que había llegado a más de medio millón de habitantes en 1537. Además, existían barreras lingüísticas que dificultaban la comunicación efectiva de ideas religiosas. Finalmente, la población indígena, aunque relativamente numerosa, estaba muy dispersa a lo largo de un territorio extenso. Las poblaciones muiscas estaban muy apartadas de los centros españoles como Tunja o Villa de Leiva. Sin embargo, al examinar cuidadosamente la naturaleza de la campaña de evangelización y de quienes la lideraban, queda claro que la presencia de alguien como Vicente de Requejada no es tan extraña como se podría pensar.

\section{La introducción del cristianismo}

En el verano de 1524, sólo tres años después de la victoria militar de Cortés sobre los aztecas y la caída de Tenochtitlán, un grupo de doce franciscanos, liderado por Martín de Valencia, arribó a la Nueva España. Estos "Doce Apóstoles", como llegaron a conocerse, caminaron descalzos 270 millas por el difícil terreno entre la

\footnotetext{
${ }^{8}$ John Frederick Schwaller, The Church and Clergy in Sixteenth-Century Mexico (Albuquerque: University of New Mexico Press, 1987), 75.
} 
población costera de Vera Cruz y Ciudad de Méjico. El arduo viaje duró más de un mes. La conquista espiritual de Méjico había empezado bajo el signo de un renovado fervor apostólico, y era liderada por un pequeño grupo de misioneros altamente calificados, dedicados y entusiastas ${ }^{9}$. Las décadas que siguieron a la llegada de los franciscanos se conocieron luego como los "años dorados" de la conquista espiritual, un periodo caracterizado por escenas de euforia que de acuerdo con John Leddy Phelan, solo se podrían comparar con los días gloriosos de la Iglesia preconstantina ${ }^{10}$.

Sin embargo, aquellas prósperas escenas, justificadas o no, no se repetirían en la Nueva Granada. Tampoco existieron grandes gestos simbólicos para inaugurar la llamada "conquista espiritual" de los indígenas muiscas. De hecho transcurrió un tiempo relativamente largo entre las fundaciones de los primeros pueblos españoles en 1537 y el lanzamiento de las primeras campañas misioneras. Solamente hasta que se instituyó la Audiencia de Santa Fe en 1550, más de una década después de la conquista, fueron establecidas formalmente las primeras órdenes religiosas en la Nueva Granada. Ese año marcó la llegada tanto de los dominicos como de los franciscanos. Tuvieron que pasar otros veinticinco años para que los agustinos establecieran su primer monasterio. Los jesuitas, cuya presencia numérica en la provincia de Tunja nunca igualó la de las otras tres órdenes, no arribaron sino hasta $1599^{11}$.

La llegada de los primeros misioneros a Tunja no trajo consigo el triunfalismo que caracterizó a las primeras décadas del "encuentro espiritual" en la Nueva España. Por el contrario, las evidencias sugieren que la atmósfera en la Nueva Granada era completamente opuesta a la euforia general que se propagó en las primeras campañas al territorio mejicano. Es más, los primeros religiosos de la Nueva Granada no parecen haber tenido las mismas cualidades que los de la Nueva España ${ }^{12}$. En una carta de 1553, Fray Juan de Soto condenaba las actividades de los misioneros de la región.

\footnotetext{
${ }^{9}$ Ver John Leddy Phelan, The Millenial Kingdom ofthe Franciscans, 2a. ed. (Berkeley: University of California Press, 1970), 48.

${ }^{10}$ Ibid.,48.

$11 \quad$ Luis Carlos Mantilla, Los Franciscanos en Colombia (Bogotá: Kelly, 1984), 1:58.

${ }^{12}$ Schwaller, Church and Clergy, 75.
} 
Exhortaba a que Felipe II hiciera algo para prevenir que tales "idiotas" llegaran a la Nueva Granada, pues no sabían ni leer ni rezar, y sus únicas actividades eran comprar, vender y sentar mil malos ejemplos ${ }^{13}$. Jerónimo de San Miguel se quejaba de que los dominicos eran unos miserables y que sólo querían partir hacia las más exóticas (y rentables) tierras del Perú ${ }^{14}$. El oidor Tomás López reportaba en 1557 que los primeros frailes de la Nueva Granada habían causado tantos escándalos que la situación sería mejor si no hubieran dado su errada instrucción religiosa a los indígenas ${ }^{15}$.

Más de cuarenta años después, el arzobispo Bartolomé Lobo Guerrero expresó preocupaciones similares y condenó el descuido y la negligencia de los clérigos de la Nueva Granada. Lamentaba el triste legado del clero y culpaba tanto a los clérigos seglares como a los regulares por la persistencia de la idolatría. Sugería además que los muiscas eran tan idólatras entonces como lo habían sido seis décadas atrás, antes de la llegada de los españoles ${ }^{16}$. "La tierra clama por remedio", escribió en una carta de 1598 al Rey ${ }^{17}$. El arzobispo no intentaba esconder su angustia; en la misma carta, enfatiza las cuatro razones que según él, explicaban el fracaso de la campaña misionera en la Nueva Granada, tres de las cuales se centraban en las fallas y defectos del clero. En primer lugar, según el arzobispo los religiosos se negaban obstinadamente a aprender lenguas nativas. Segundo, sentaban tan mal ejemplo a los indígenas, que se causaron daños irreparables a ellos mismos y al mensaje que debían estar predicando. Finalmente, su falta de cultura y educación eran tan marcadas, que no sólo los nativos recibían una pésima instrucción; al mismo tiempo, la población española de la Nueva Granada corría el riesgo de caer en el ocio, la decadencia y la ignorancia ${ }^{18}$.

\footnotetext{
${ }^{13}$ Juan Friede, Fuentes Documentales para la Historia del Nuevo Reino de Granada (Bogotá: Biblioteca Banco Popular, 1975), 2:28. ${ }^{14}$ Ibid., 1:36.

${ }^{15}$ Ibid., 3:183.

${ }^{16}$ Archivum Romanum Societatis Iesu, Roma (en adelante citado como ARSI), Novi Regni et Quito, 14, fol. 6v.

${ }^{17}$ ARSI, Novi Regni et Quito, 14, fol. 7r.

${ }^{18}$ ARSI, Novi Regni et Quito, 14, fol. 7r. La cuarta razón para la persistencia de la idolatría, de acuerdo con Lobo Guerrero, surgía del tosco tratamiento que los encomenderos daban a los indígenas. Respecto al clero, la solución de Lobo Guerrero consistía en apelar a la corona para que los jesuítas liderasen la campaña de conversión de nativos en la Nueva Granada. Aunque el arzobispo obtuvo su deseo, la llegada de apenas un puñado de jesuitas en 1599 no parece haber creado ningún cambio dramático en la conquista espiritual de los muiscas.
} 
Críticas similares se pueden encontrar a lo largo de los registros coloniales del siglo XVII y principios del XVII, con fuertes condenas tanto a los sacerdotes seglares como a los regulares. Miembros de ambos grupos fueron descritos como hombres perezosos, venales, arrogantes $\mathrm{y}$, sobre todo, ignorantes en materias de $\mathrm{fe}^{19}$. A primera vista parece que el fracaso de la conquista espiritual de la Nueva Granada se podría explicar por las faltas del clero de la región. Sin embargo, enfocarse demasiado en los errores de un pequeño grupo de sacerdotes y frailes que se responsabilizaron de la conversión de más de medio millón de almas en el territorio muisca, niega la posibilidad de reconocer los múltiples obstáculos que los religiosos tuvieron que enfrentar, y las diversas propuestas y políticas generadas para superarlos.

\section{Los obstáculos}

Hay que recordar, además de los cáusticos ataques dirigidos a los primeros misioneros de la Nueva Granada, que los informes tempranos a la Corona y al Consejo de Indias enfatizaban los enormes obstáculos que el clero debía enfrentar, entre ellos la necesidad de establecer formas efectivas para comunicar los misterios de la fe. Una verdadera instrucción religiosa requería un sofisticado conocimiento de las lenguas nativas (en este caso el chibcha), algo que pocos sacerdotes, en su mayoría mestizos, lograron obtener ${ }^{20}$. En 1580, Pedro de Zorrillo, un oidor de la Audiencia de Santa Fe, informaba al Consejo de Indias que los nativos de la Nueva Granada eran tan ignorantes entonces (en materias espirituales) como antes de la conquista. De acuerdo con Zorrillo, esta ignorancia era producto del carácter obstinado de los sacerdotes locales, quienes se negaban a aprender las lenguas de los nativos. Pocos de ellos hablaban chibcha y por ello se veían forzados a enseñar la doctrina en español o en algunos casos en un latín que los indígenas repetían "como papagayos". ${ }^{21}$

\footnotetext{
${ }^{19}$ A continuación de su extensa inspección de la provincia en 1635 y 1636, Juan de Valcárcel enumeraba seis razones por las cuales pensaba que los indígenas de Tunja no habían recibido la fe católica. La sexta, que era el mayor obstáculo por superar, era el mal ejemplo que brindaba la población española, incluido el clero. Ver Ulises Rojas, Documentos Inéditos para la Historia de Boyacá y Colombia (Tunja: Academia Boyacense de Historia, 1991), 1:106.

${ }^{20}$ D. A. Brading, The First America (Cambridge: Cambridge University Press, 1991), 106.

${ }^{21}$ Juan Manuel Pacheco, La Evangelización del Nuevo Reino, Siglo XVI, vol. 13, t. 1 de Historia Extensa de Colombia (Bogotá: Lerner, 1971), 483.
} 
A principios de la década de 1580 a 1590, la Audiencia intentó remediar la situación, obligando a todo el clero a asistir a clases de chibcha. En 1582, un sacerdote llamado Gonzalo Bermudez fue llamado para instruir a los religiosos de la Nueva Granada. Las clases empezaron en mayo de ese año y se dictaban en la Catedral de Santa Fe de Bogotá $^{22}$. Sin embargo, no parecen haber surtido el efecto deseado; en 1598, Bartolomé Lobo Guerrero, el nuevo arzobispo de Santa Fe, repitió lo que Zorrillo ya había dicho, afirmando que el clero se negaba obstinadamente a aprender chibcha, y que esta era la razón primordial para el lamentable estado de los asuntos religiosos en la región ${ }^{23}$.

El mismo año en que Bermudez fue llamado a dar clases de chibcha al clero, los superiores dominicos, franciscanos y agustinos lanzaron comunicados en los que declaraban que tal enseñanza lingüística era innecesaria e inútil. Muchos de los religiosos se oponían con vehemencia al establecimiento de una cátedra de chibcha. Afirmaban que esta lengua no era lo suficientemente compleja como para enseñar con precisión los misterios y dogmas de la fe cristiana.

Tales aserciones fueron desmentidas en más de una ocasión. El arzobispo Lobo Guerrero consideraba que eran simples excusas de un clero demasiado perezoso para hacer el más mínimo esfuerzo de aprender la lengua ${ }^{24}$. Además, reconocía que se trataba de una labor ardua, pero estaba convencido de que el reto podría ser superado con la diligencia y dedicación que, supuestamente, son propias de los frailes $^{25}$. A pesar de las exhortaciones de Lobo Guerrero, los intentos por convencer a los religiosos de la importancia de aprender lenguas nativas fueron infructuosos. Es más, los religiosos ampliaron sus críticas anteriores respecto a las limitaciones inherentes a la lengua chibcha e insistieron en que las clases de Bermudez tendrían un valor limitado debido a la diversidad lingüística de la región.

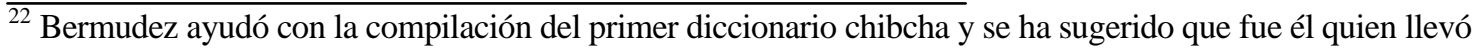
a cabo una traducción del catecismo a esta lengua indígena. Continuó enseñando chibcha hasta su muerte en abril de 1625. ${ }^{23}$ ARSI, Novi Regni et Quito, 14, fol. 6v.

${ }^{24}$ ARSI, Novi Regni et Quito, 14, fols. 6v-7r.

${ }^{24}$ Ibíd.
} 


\section{FRONTERAS}

De acuerdo con los superiores de las tres órdenes, el chibcha que hablaban los nativos de Bogotá era distinto del que hablaban en Tunja. Aceptaban que Bermúdez hablaba muy bien la lengua, pero agregaban que solamente dominaba el dialecto del Valle de Ubaqué (en la provincia de Santa Fe), y que sus enseñanzas ignoraban completamente los dialectos que se hablaban en otras regiones ${ }^{26}$. Además, los superiores afirmaban que ningún religioso de Santa Fe o de Tunja, incluyendo a Bermúdez, comprendía el chibcha lo suficientemente bien como para traducir los misterios y verdades de la $\mathrm{fe}^{27}$. Según ellos, era mucho más importante que el clero de la Nueva Granada estudiara Teología y recibiese clases de latín ${ }^{28}$.

En una carta fechada el 25 de agosto de 1606, el presidente de la Audiencia, Juan de Borja, expresaba sus propias preocupaciones respecto al tema. Borja estaba del lado del arzobispo, al afirmar que el mayor obstáculo para la conversión efectiva de los indígenas era la imposibilidad de que el clero brindara una instrucción religiosa en chibcha. Aplaudía la cooperación de los sacerdotes y frailes que trabajaban juntos para producir traducciones al chibcha del Credo, el Padre Nuestro y de un breve Catecismo. Sin embargo, las traducciones no complacían a todo el mundo y aún existía una fuerte hostilidad ante el mandato del arzobispo según el cual toda enseñanza religiosa debía impartirse en lengua nativa. El presidente Borja escribió que el debate no se limitaba únicamente a los miembros del clero; al parecer todos en Santa Fe tenían una opinión al respecto y se oían acaloradas discusiones sobre el tema a lo largo de las plazas y calles de la población ${ }^{29}$.

\footnotetext{
${ }^{26}$ Las afirmaciones respecto a la diversidad lingüística del territorio muisca encontraron apoyo en dos cronistas religiosos del siglo XVII. El franciscano Fray Pedro Simón escribió que no había ninguna lengua común en las provincias muiscas de Bogotá y Tunja; por el contrario, afirmaba Simón, los nativos de cada pueblo hablaban su propia lengua. Otro cronista de ese siglo, el mestizo Lucas Fernández de Piedrahíta, sugiere igualmente que no había una lengua común en el territorio muisca, algo que él atribuía a la fragmentación política (por la estructura de cacicazgos) que existía antes de la conquista. Infortunadamente los archivos no aclaran la materia. Era común en las visitas coloniales ver a un mismo individuo servir como lengua o traductor a lo largo de toda una inspección, sin referencia alguna a dialectos regionales. Sin embargo, también es posible encontrar ejemplos que ofrecen alguna credibilidad a los argumentos de los religiosos: en 1621. Francisco Beltrán, un mestizo que vivía en Guatavita (provincia de Santa Fe), afirmaba que no podía servir como intérprete en la inspección de Antonio de Obando a Tenza (provincia de Tunja) ya que la lengua era diferente y él no podía entenderla. Ver AGN, Visitas Boyacá, 15, fol. 195r (1621).

${ }^{27}$ Humberto Triana y Antorveza, Las Lenguas Indígenas en la Historia Social del Nuevo Reino de Granado (Bogotá: Instituto Caro y Cuervo, 1987), 265.

${ }^{28}$ Ibid., 180.

${ }^{29}$ ARSI, Novi Regni et Quito, 17, fols. 48r-48v.
} 
El conflicto sobre la lengua chibcha fue, sin duda, parte de una disputa más amplia entre los oficiales seglares y las órdenes religiosas respecto a la jurisdicción sobre la población nativa, una disputa que claramente no estaba restringida a la Nueva Granada $^{30}$. Más aún, tal controversia se intensificó en la década de 1570 a 1580 , luego de la Ordenanza del Patronazgo de 1574 y el intento de la Audiencia por cumplir con las órdenes de España. En 1578 el Consejo de Indias lanzó un decreto real a cada una de sus diócesis en el cual prohibían el nombramiento de un párroco que no comprendiese la lengua de sus feligreses. Dos años después, en 1580 se enviaron órdenes adicionales a las Audiencias de Charcas, Quito y Santa Fe para que estableciesen escuelas que enseñaran las lenguas nativas. Además de la institución de estas nuevas escuelas, el Consejo incluía cuatro órdenes más. Primero, se estipulaba que en adelante ningún sacerdote podría ser ordenado sin haber estudiado al menos un año de lenguas nativas. Segundo, aquellos que tuviesen un mejor dominio de tales lenguas tendrían preferencias en términos de los beneficios otorgados. Además, ningún sacerdote, ya fuese español o de otra procedencia, podría ser llamado a trabajar en parroquia alguna sin haber demostrado una comprensión mínima del lenguaje local. Finalmente, se ordenaba que al término de un año todos los sacerdotes servidores en la provincia debían tomar un examen para demostrar sus habilidades lingüísticas ${ }^{31}$.

Felipe III reiteró luego las órdenes de su padre: todos los religiosos debían ser examinados para asegurar que comprendiesen el lenguaje de sus feligreses. En 1605 un nuevo conjunto de exámenes competitivos fue organizado en Nueva Granada bajo la supervisión del presidente de la Audiencia, Juan de Borja. Los resultados de los exámenes perjudicaron a los dominicos y especialmente a los franciscanos. El arzobispo de Santa Fe, Bartolomé Lobo Guerrero, se vio obligado a destituir a todos los doctrineros incapaces de predicar y oír confesión en chibcha. En la provincia de Tunja, los franciscanos perdieron su autoridad sobre las doctrinas de Tibaná, Icabuco, Chiribí, Busbanza, Ocavita, Tupachoque, Beitivá, Tibasosa y Tinjacá. Los dominicos fueron expulsados de las doctrinas de Onzanga y Ramiriquíi ${ }^{32}$.

\footnotetext{
${ }^{30}$ Para tener una visión concisa sobre este conflicto en la Nueva España, ver Robert Charles Padden, "The Ordenanza del Patronazgo of 1574: An Interpretative Essay," The Americas 12, (1956): 333-54.

${ }^{31}$ Fray Alberto Lee López, "Clero Indígena en el Arzobispado de Santa Fe en el Siglo XVI," Boletín de Historia y Antigüedades 50 (1963), 23-24.

${ }^{32}$ Triana, Las Lenguas Indígenas, 456, 457.
} 
Hay que señalar, sin embargo, que no todos los religiosos compartían el escepticismo expresado por franciscanos, dominicos y agustinos. El jesuita José Dadey por ejemplo, quien llegó a la Nueva Granada desde Méjico a finales del siglo XVI. consideró el chibcha como una lengua fácil de aprender y sugirió con gran arrojo que cualquiera podría dominarla luego de un año de estudio ${ }^{33}$. En 1604 completó su Arte y Gramática de la Lengua Muisca, un ambicioso proyecto que muchos miembros de la Iglesia consideraban imposible pues pensaban que el chibcha era una lengua "horrorosa", difícil de pronunciar y carente de un vocabulario amplio ${ }^{3 " .}$ Dadey por su parte, presumía haber esbozado exitosamente algunas traducciones al chibcha de ciertas oraciones y de un breve catecismo.

A pesar de los esfuerzos de este jesuita, y de aquellos de sacerdotes como Gonzalo Bermúdez, muy pocos religiosos aprendieron chibcha ${ }^{35}$. Pronto el entusiasmo por el lenguaje y la campaña de instrucción a los clérigos empezaron a decaer. Mientras los años pasaban y la población nativa continuaba su devastador declive, los intentos por brindar instrucción religiosa en chibcha disminuyeron dramáticamente. El lenguaje mismo estaba cambiando. Incluso Dadey observó que el chibcha estaba empezando a perder algo de su "fuerza y autenticidad" a inicios de siglo XVII ${ }^{36}$. Otros hechos sugieren también que el lenguaje hablado por los muiscas había cambiado significativamente a finales del siglo XVI; sin embargo, la transición era mucho más evidente en (y cerca de) las poblaciones españolas más importantes como Santa Fe y Tunja, y entre los indígenas que al trabajar como mercaderes i criados, tenían un contacto permanente con los españoles ${ }^{37}$. El mayor acercamiento

\footnotetext{
${ }^{33}$ Ibíd.,418.

${ }^{34}$ Ibid.,418.

${ }^{35}$ De acuerdo con el presidente de la Audiencia, Juan de Borja, para 1606 sólo un puñado de religiosos aprendió la lengua. Eran los franciscanos Luis de Mejorada y Nicolás de Troya; Gaspar de Alvarado y Vicente Mallol de la orden de San Agustín; los dominicos Juan de Avalos y Bernardo de Lugo, y los jesuítas Juan Antonio, Martín Vázquez, el padre José (probablemente José Dadey) y Juan Bautista. Borja también menciona a los sacerdotes seglares Pedro Gutiérrez y Gonzalo Bermúdez, al igual que al encomenderc Diego Romero de Aguilar, y a los intérpretes de la Audiencia Juan de Lara y Juan Sepúlveda, como los más capaces para traducir la doctrina al chibcha.

${ }^{36}$ Triana, Las Lenguas Indígenas, 167.

${ }^{37}$ Aunque no se han descubierto suficientes evidencias para apoyar tal conclusión, los cambios lingüísticos resultaron probablemente de un "préstamo masivo" de sustantivos españoles, un proceso descrito de manera convincente en el excelente estudio sobre la sociedad Nahua bajo el imperio colonial de James Lockhart. Ver James Lockhart, The Nahuas After the Conquest (Stanford: Stanford University Press, 1992).
} 
entre españoles e indígenas empezaba a producir una tercera lengua, una mezcla entre español y chibcha que un contemporáneo denominó, despectivamente, "gitano"

A pesar de los esfuerzos de algunos clérigos de la región, parece que la mayor parte de la instrucción religiosa en la Nueva Granada durante el primer periodo colonial se dio en español o en latín; a medida que la población nativa declinaba, se recalcó menos la necesidad de que el clero tuviese que aprender chibcha. La legislación real de mediados del siglo XVII simplemente confirma esta realidad. Un decreto expedido en abril de 1641 ordenaba que el chibcha no debía ser utilizado en adelante, y que todos los indígenas debían aprender español. El decreto justificaba la decisión de forzar a los muiscas a aprender castellano haciendo referencia a las prácticas del Inca: "... pues bien, ¿no es cierto que en tiempos del Inca era obligatorio para todos [sus vasallos] aprender quechua? Y así lo hicieron" ${ }^{39}$.

Aun así, el lenguaje fue sólo uno entre muchos obstáculos que dificultaron la temprana campaña misionera en la Provincia de Tunja.

Otro obstáculo serio era, sencillamente, el número de personas disponibles para la empresa. Aunque no sabemos cuál fue el número exacto de religiosos presentes en Tunja en el siglo XVI y principios del XVII, las evidencias sugieren que las cifras

\footnotetext{
${ }^{38}$ Triana, Las Lenguas Indígenas, 167. La mayoría de los indígenas de la provincia de Tunja siguió hablando chibcha durante el siglo XVI. Juan Villamarín descubrió que, para la provincia de Santa Fe, la mayoría de los indígenas que testificaron durante las visitas sólo podían hablar chibcha y necesitaban un traductor a la hora de dar declaraciones. Ver Juan A. Villamarín, "Encomenderos and Indians in the Formation of Colonial Society in the Sabana de Bogotá, Colombia, 1537-1740" (Disertación para optar al título de doctorado, Brandeis University, 1972), 122. De acuerdo con la Descripción de Tunja de 1610, "El español se habla generalmente en esta ciudad, excepto entre los indios, quienes hablan sus propias lenguas; y aquellos que sirven a los españoles hablan español, algunos mejor que otros, hasta que se hacen ladinos". Ver Luis Duque Gómez, Tribus Indígenas y Sitios Arqueológicos, vol. 1, t. 2 de Historia Extensa de Colombia, 545. ${ }^{39}$ Triana, Las Lenguas Indígenas, 231. Una de las razones para el cambio de política a mediados del siglo XVII era el declive demográfico de la población nativa de Tunja, que en los inicios de la conquista excedía los 200.000 habitantes. Sin embargo, durante el siguiente siglo la población indígena sufrió una fuerte caída, llegando a los 65.000 individuos para 1602. A diferencia de otras regiones del Nuevo Mundo, que vieron indicios de una recuperación demográfica para la década final del siglo XVI, la población indígena de Tunja siguió disminuyendo, alcanzando los 50.000 habitantes cuando Juan de Valcárcel realizó su inspección de la provincia entre 1635 y 1636. Para un sondeo detallado de los cambios demográficos en la provincia de Tunja entre 1537 y 1636, ver J. Michael Francis, "The Muisca Indians Under Spanish Rule" (Disertación para optar al título de doctorado, University of Cambridge, 1998), 97-171.
} 
nunca fueron muy altas ${ }^{40}$. Por ejemplo, en 1560 los dominicos acababan de construir su primera residencia permanente en Tunja. El monasterio era pequeño y no podía albergar más de ocho monjes ${ }^{41}$. Aun así, los dominicos doblaban la presencia franciscana, compuesta por cuatro frailes que compartían una casa con techo de paja en las afueras del pueblo ${ }^{42}$. Diez años después, en 1570, Juan López de Velasco reportaba que había dos monasterios en Tunja: uno dominico y otro franciscano. Sólo había cuatro frailes residentes en cada uno, y existían otros veinte frailes dispersos por las diferentes doctrinas de la provincia ${ }^{43}$.

En 1607, a excepción del recién fundado monasterio agustino, las cosas habían cambiado poco. El número de dominicos se incrementó a 30, pero sólo había 20 franciscanos y 6 agustinos en toda la provincia ${ }^{44}$. Para mediados del siglo XVII, sólo los agustinos habían incrementado sus filas significativamente, pasando de 6 en 1607 a 21 en 1651. El número de dominicos se había reducido a 25, una cifra igual al total de franciscanos. Además de ellos, había 9 jesuitas ${ }^{45}$.

\footnotetext{
${ }^{40}$ De acuerdo con Alberto Lee López, 129 sacerdotes fueron ordenados en Nueva Granada en el siglo XVI. Lee López y Humberto Triana han identificado a 24 monjes que sirvieron en la provincia de Tunja. El únicc español entre ellos era Juan de Leguizamón e incluso a él lo llevaron a muy temprana edad. Al menos 11 de los sacerdotes eran mestizos: Juan de Figueredo, Gonzalo García Matamoros, Martín Gaitán, Andrés Ortíz de Godo, Martín Partearrollo, Pedro Rincón, Alonso Rodríguez Parra, Hernando de Rojas, Juan Vázquez. Esteban Zambrano y Juan García Matamoros. Se sabe que Lorenzo Martín de Benavides, Gonzalo Velasco. Juan Bravo de Guzmán, Cristóbal Sanabria y Rodrigo Vargas eran criollos, pero no hay información respecto a los otros siete sacerdotes de Tunja. Ver Fray Alberto Lee López, "Clero Indígena," 29-56, y Triana, Las Lenguas Indígenas, 144-46. No es del todo claro dónde recibieron su educación los mestizos; sin embargo, para mediados de la década de 1550 a 1560, muchos mestizos asistían a clases en casa de Diego de Aguila en Tunja. Entre sus pupilos se contaban Sebastián Ropero, Diego Carvajal, Antón de Rojas, Alonso de Silva. Miguel de Partearroyo, Cristóbal de Sanabria y Andrés Camacho. Otro de sus estudiantes era Martín de Requejada, el hijo mestizo de fray Vicente de Requejada. Ver Ulises Rojas, El Cacique Turmequéy su Época (Tunja: Imprenta Departamental de Boyacá, 1965), 8.

${ }^{41}$ Hermes Tovar Pinzón, No Hay Caciques ni Indios (Barcelona: Sedai, 1988), 43.

${ }^{42}$ Ibid., 43.

${ }^{43}$ Juan López de Velasco, Geografía y Descripción Universal de las Indias, Biblioteca de Autores Españoles, ed. Don Marcos Jiménez de Espada, n. 248 (Madrid: Atlas, 1971), 184-85. También para esta época (1570). el encomendero Francisco Salguero y su esposa Juana Macías de Figueroa fundaron el primer convento de Tunja, Santa Clara Real.

${ }_{45}^{44}$ AGI, Santa Fe, 231, n. 60.

45 Julián B. Ruiz Rivera, Encomienda y Mita en Nueva Granada en el Siglo XVII (Sevilla: Escuela de Estudios

Hispanoamericanos, 1975), 116.
} 
La escasez de monjes significaba que la mayoría de los religiosos eran responsables por el bienestar espiritual de varios pueblos a la vez, muchos de los cuales se encontraban dispersos en territorios muy extensos. Por ello, el clero se quejaba constantemente de la imposibilidad de supervisar efectivamente las actividades de su rebaño. Algunos sacerdotes afirmaban que dos o tres días después de dejar un pueblo, los indígenas volvían a sus antiguos hábitos y a conversar con el demonio según sus costumbres $^{46}$. Otros advertían que los indígenas desaparecían a menudo de sus pueblos, afirmando que tenían que trabajar los campos, cuando en realidad iban a adorar a sus ídolos ${ }^{47}$. Como es de esperarse, había peticiones constantes por la presencia de más sacerdotes en la región ${ }^{48}$.

Aunque los informes tempranos a la Corona muestran algunos de los obstáculos que enfrentaron los primeros religiosos en Tunja, sólo hasta 1560, con los registros que surgieron de la visita general de Tomás López en ese año, es posible obtener un primer vistazo al estado en que se encontraba la campaña misionera en esta población. Uno de los principales objetivos de la visita de López era medir el "bienestar" espiritual de la población indígena y por ello el tema religioso domina los textos. López descubrió en su extensa inspección, para su desencanto, que la gran mayoría de los indígenas de Tunja sólo habían recibido una débil y descuidada supervisión religiosa. De hecho, encontró que algunas comunidades habían sido olvidadas del todo. Los nativos de la encomienda de Juan de Torres en Iza, le informaron que nunca habían visto a un sacerdote en su pueblo ${ }^{49}$. También descubrió que solamente unos pocos pueblos de Tunja tenían la presencia permanente de un religioso. El Capitán Bechaque, un indígena de la encomienda de Andrés López de Galarza en el Cocuy, le informó al visitador que cuando Pedro de Colmenares era el encargado de esa misma encomienda, un sacerdote había permanecido allí por un mes. Bechaque agregó que esa fue la unica vez en que un fraile estuvo presente; desde entonces los indígenas del Cocuy no habían recibido visitas de otros religiosos y morirían sin haberse convertido al cristianismo ${ }^{50}$. En su defensa, Andrés López

\footnotetext{
${ }^{46}$ Friede, Fuentes Documentales, 3:357.

${ }^{47}$ AGI, Patronato, 27, r. 34, carta del fiscal Francisco Guillen Chaparro, fol. lv.

${ }^{48}$ Friede, Fuentes Documentales, 3:252.

${ }^{49}$ AGN, Visitas Boyacá, 13, fol. 206.

${ }^{50}$ AGN, Visitas Boyacá, 13, fol. 295. Antes en esa misma inspección, el cacique del Cocuy también testificó que un sacerdote había estado presente por un periodo cercano a un mes; sin embargo, afirmó que la visita había ocurrido mientras Andrés López de Galarza era el encomendero.
} 
de Galarza le dijo al visitador que él tomaba muy en serio sus obligaciones como encomendero y que había hecho los mayores esfuerzos por garantizar el bienestar espiritual de sus indígenas. De hecho, en varias ocasiones había solicitado la ayuda del Obispo y de los dominicos, pero la falta de religiosos en la región y las treinta leguas que separaban al Cocuy de Tunja hacían que sus peticiones fueran constantemente negadas $^{51}$.

Incluso las comunidades muiscas que estaban localizadas en las proximidades de Tunja encontraron dificultades para obtener una instrucción religiosa estable. Los indígenas de Suta, Tuniquirá (Banganique), Socotá y Cibatá le informaron al visitador Tomás López que desde la llegada de los españoles, 23 años atrás, los sacerdotes sólo habían estado presentes en sus pueblos por periodos cortos de tres o cuatro meses $^{52}$. Los encomenderos de Tunja presentaron protestas similares a las que el visitador López había oído en el Cocuy. Antonio de Santana se quejaba de las dificultades que había tenido que enfrentar para conseguir un sacerdote permanente para su encomienda de Suta. En la época de la inspección de López. Santana seguía su búsqueda. Entre tanto, había llamado a un hombre español (probablemente uno de sus trabajadores) para que diera una instrucción cristiana básica a los indígenas ${ }^{53}$. Una situación semejante se presentaba en Chibatá donde el encomendero, Pedro de Rivera afirmaba que por la insuficiencia de sacerdotes y frailes en la provincia, él y su hijo se habían visto obligados a convertir a los indígenas ${ }^{54}$. Tal declaración había sido confirmada de antemano por uno de los capitanes de Chibatá, quien informó que cuando los nativos de la encomienda se enfermaban, era el encomendero quien los "hacía cristianos". ${ }^{55}$ En otras palabras, el mismo Pedro de Rivera los bautizaba. Algunos encomenderos iban más allá para asegurar que sus indígenas recibieran alguna instrucción religiosa. Martín Sánchez Ropero, encomendero de Socotá, testificó que había confiado la enseñanza religiosa de los nativos de su encomienda a algunos indios ladinos ${ }^{56}$.

\footnotetext{
${ }^{51}$ AGN, Visitas Boyacá, 13, fol. 300.

${ }^{52}$ AGN, Visitas Boyacá, 13, fols. 200, 214, 265, 316. (Nota: Tuniquirá probablemente formaba parte de Banganique, la encomienda de Juan de Orozco. Para 1562, cuando Angulo de Castejón llevó a cabo su detallada inspección de la provincia, ya no había mención alguna de Tuniquirá como una encomienda aparte.)

${ }^{53}$ AGN, Visitas Boyacá, 13, fol. 201.

${ }^{54}$ AGN, Visitas Boyacá, 13, fol. 270.

${ }^{55}$ AGN, Visitas Boyacá, 13, fol. 269.

${ }^{56}$ AGN, Visitas Boyacá, 13, fol. 317.
} 
Los habitantes de Turmequé y Boyacá se contaban entre los pocos que recibían una supervisión religiosa formal por largo tiempo, pero incluso en Turmequé, el tercer pueblo de la región con cerca de 1500 indígenas tributarios en 1560, los nativos apenas empezaron a recibir una instrucción religiosa uno o dos años antes de la visita de Tomás López. Anteriormente, la mayoría de ellos moría "sin ser cristianos" (es decir, sin haber sido bautizados). La situación era similar en Boyacá, otro pueblo densamente poblado, con más de quinientos indígenas tributarios ${ }^{57}$ :

"... desde que Diego de Partearroyo fue nombrado encomendero [de Boyacá] hemos recibido casi tres años de instrucción religiosa de un fraile dominico; pero desde la llegada de la epidemia de viruela (en 1558) no ha habido nadie [que brinde instrucción religiosa], y no había ningún sacerdote antes de la llegada del dominico ..."

Sin embargo, incluso en pueblos como Turmequé o Boyacá, es muy improbable que se haya presentado una conversión seria y significativa de los indígenas. Por ejemplo, de acuerdo con el testimonio de un cacique "cristiano", un notario apuntó: "[el testigo] no llevó a cabo el juramento porque, aunque afirmó ser cristiano, aparentemente no entendía lo que eso quería decir" ${ }^{\prime 59}$. Observaciones similares se presentaron durante toda la inspección de 1560, por lo cual pronto López comprendió con gran dolor, que los logros de los primeros misioneros y sacerdotes no eran más que un breve compendio de bautismos.

Escenas parecidas a las descritas en la visita de López se presentaron once años después cuando Juan López de Cepeda llevó a cabo su inspección general de la

\footnotetext{
${ }^{57}$ Basado en testimonios de un capitán llamado Alonso y el cacique de Turmequé, AGN, Visitas Boyacá, 13, fols. 235r, 236r-237r.

${ }^{58}$ AGN, Visitas Boyacá, 13, Testimonio de Don Pedro, Indio Principal de Boyacá, fol. 306. El testimonio resulta sorprendente si se considera el largo tiempo que Diego de Partearroyo fue encomendero de Boyacá. Tuvo la encomienda por casi cuatro décadas, desde 1542-1543 hasta 1583, en los cuales los indígenas sólo tuvieron tres años de educación religiosa continua. La referencia a la epidemia de viruela es importante pues duró dos años (1558-1560) e hizo manifiesta la escasez de clérigos en la provincia.

Muchos de los indígenas que testificaron ante López le informaron que sólo habían visto a los clérigos durante la epidemia, cuando se vieron obligados a moverse de población en población en un intento desesperado por convertir a la mayor cantidad posible de indígenas. Ver AGN, Visitas Boyacá, 13, fols. 212,236v-237r y Friede, Fuentes Documentales, 3:356.

${ }^{59}$ AGN, Visitas Boyacá, 13, fol. 306.
} 
provincia. Nuevamente los indígenas testificaron que los sacerdotes trabajaron en sus pueblos por uno o dos meses y luego desaparecieron. Debido a la ausencia de un sacerdote, los nativos se reunían a rezar con los encomenderos o con otros cristianos que residían en sus poblaciones. La insuficiencia de religiosos era evidente. Por ejemplo, cuando le preguntaron si existían indígenas cristianos en su pueblo, el cacique de Pisba respondió que no, ya que no había nadie que los pudiera "hacer cristianos" 60 .

No hay forma de conocer el efecto que tuvieron las primeras campañas misioneras en las creencias religiosas de la población nativa de Tunja. Ninguno de los indígenas testificó sobre tal tema. Sin embargo, sería poco realista esperar que los esfuerzos de los primeros eclesiásticos llevaran a un rápido desplazamiento de la religión muisca, un argumento que algunos historiadores sostienen y que nunca ha sido cuestionado seriamente por quienes estudian esta cultura ${ }^{61}$. Esto no quiere decir que los indígenas de Tunja estuviesen inclinados por naturaleza a no creer en el cristianismo; muestra más bien que durante las primeras campañas misioneras, pocos indígenas de la Tunja del siglo XVI tuvieron un contacto cotidiano con la nueva religión. Para la mayoría de la población de este centro, la conversión temprana a la cristiandad probablemente consistía apenas en ser bautizado, recibir un nombre cristiano y, en palabras de Susan Ramírez, recibir un "conocimiento superficial" sobre una nueva deidad ${ }^{62}$.

\footnotetext{
${ }^{60}$ AGN, Visitas Boyacá, 29, fols. 80r-80v.

${ }^{61}$ Por ejemplo, María Imelda López Avila afirma que la religión muisca desapareció rápidamente porque la cercana supervisión de los religiosos españoles les negaba a los indígenas la posibilidad de asistir a sus santuarios anteriores a la conquista, o de seguir practicando sus antiguos rituales. Ver María Imelda López Avila, Historia y Cultura Populares, ed. Pablo Mora Calderón y Amado Guerrero Rincón (Tunja: Centro de Investigación de Cultura Popular del ICBA, 1989), 80.

${ }^{62}$ Susan Ramírez, The World Turnea. Upside Down: Cross-Cultural Contact and Conflict in Sixteenth-Century Peru (Stanford: Stanford University Press, 1996), 134. El testimonio de una indígena que fue interrogada por Jusepe de Valtierra en 1598 sirve de ejemplo para esto. Cuando le pidieron que diese su nombre ella respondió que no era cristiana, aunque enmendó rápidamente su respuesta y afirmó que sí lo era. Valtierra le preguntó entonces: "¿Si en verdad eres cristiana por qué acabas de negarlo?" La mujer respondió que había olvidado que se había hecho cristiana. Dijo que muchos años antes, cuando era apenas una niña, se había enfermado. Un sacerdote vino a visitarla, la bautizó, y le dio el nombre de Leonor. Ver AGN, Visitas Boyacá. 13 ,fol.312.
} 
Todos los visitadores del siglo XVI señalaban tanto la necesidad de brindar una mayor instrucción religiosa como la importancia de tener iglesias en cada pueblo. Por ejemplo, Tomás López informó que en 1560 solo había un puñado de poblaciones indígenas que contasen con una iglesia ${ }^{63}$. Más de 20 años después de la conquista, incluso la iglesia de Tunja, un edificio pequeño de ladrillo y adobe, no se había terminado ${ }^{64}$. Este hecho no es sorprendente teniendo en cuenta que la provincia tenía un número muy reducido de clérigos y habitantes españoles, y que los indígenas se encontraban muy dispersos por toda la extensa región. Aun así, la situación no había mejorado mucho cuatro décadas después, cuando Luis Enríquez inició su visita de dos años a la provincia.

En una carta fechada el 25 de abril de 1602, Enríquez informaba que de las trece encomiendas que la Corona tenía en la provincia, sólo dos contaban con iglesias apropiadas. Encontró sólo cuatro o cinco más en las otras 153 encomiendas de la región; "las iglesias restantes, que son pocas, son edificios de adobe mal construidos, con techos de paja... y son casas más pobres que las de la mayoría de los indios"65. La inspección de Enríquez reveló que aquellos pueblos con iglesias tenían grandes poblaciones tributarias; sólo Boavita (66), Suta (175), Lenguasaque (188) y Tota (115) tenían poblaciones inferiores a los 200 indígenas tributarios. Las cifras demográficas que se recogieron durante la visita indican que las encomiendas con más de 200 tributarios eran poco frecuentes. De hecho, para principios del siglo XVII, la mayoría de encomiendas tenía menos de cien tributarios. Estas encomiendas menores, algunas de las cuales estaban alejadas de cualquier población española, ofrecían pocos atractivos para los sacerdotes, quienes preferían esperar a ser llamados para empresas más lucrativas. Además, el costo de mantener a un clérigo permanente y construir una iglesia era excesivamente alto para las comunidades

\footnotetext{
${ }^{63}$ AGN, Visitas Boyacá, 13, fol. 312.

${ }^{64}$ Tovar, Caciques ni Indios, 91.

${ }^{65}$ AGI, Santa Fe, 18, r. 3, n. 11, bl. 2, fol. 1r. Un examen minucioso de la inspección de Enríquez muestra que su reporte sólo exageraba levemente. Los únicos pueblos con iglesias "decentes" eran Suta, Lenguasaque, Firavitoba, Sutatenza, Toca, Turmequé, Guacheta, Sogamoso, Chita, Boavita y Tota. Al concluir su inspección, Enríquez ordenó que se construyeran iglesias en Soracá, Ráquira, Samacá, Suta, Convitá, Cuqueita, Sáchica, Tibaquirá, Nemusa, Oicatá y Motavita. Ver AGI, Santa Fe, 18, r. 4, n. 29, fols. Ir-21r.
} 
dispersas que apenas tenían 40 o 50 tributarios ${ }^{66}$. Cuando López de Cepeda condujo su inspección de la provincia, un capitán testificó que en Moravita, un pueblo con 60 tributarios, no había "ni iglesia, ni campana, ni fraile que los instruya"67.

Los eclesiásticos se quejaron durante todo el siglo XVI y principios del XVII porque los muiscas vivían en poblaciones demasiado dispersas, y sus casas o bohíos quedaban muy apartados. Como resultado de esto, afirmaban que era virtualmente imposible que la Iglesia cumpliese con sus obligaciones exitosamente ${ }^{68}$. Tales patrones de establecimiento dificultaban que los sacerdotes mantuviesen una vigilancia cuidadosa de sus feligreses. Por ejemplo, las encomiendas de Lenguasaque y Guachetá, con una población combinada que excedía los cuatrocientos indígenas, eran administradas por un solo fraile dominico. El fraile era responsable del bienestar espiritual de más de 1.000 almas en dos pueblos distintos. Es difícil imaginar cómo un individuo podía manejar efectivamente a una población tan grande y tan dispersa. Tal obstáculo fue confirmado por un indígena noble de Guachetá, quien no pudo recordar el nombre del religioso, cuando le preguntaron por el encargado del cuidado espiritual de su pueblo ${ }^{69}$.

\footnotetext{
${ }^{66}$ En 1574 el salario anual de un sacerdote en la Nueva Granada se componía de: (1) cincuenta maravedí; (que debían provenir del tributo anual), (2) doce fanegas de trigo, (3) veinte fanegas de maíz, (4) tres cerdos. (5) veinte ovejas, (6) una carga de papas por semana (que debía provenir del cacique y los indios sujetos a él ) (7) tres pollos cada semana, excepto durante la Cuaresma (que también debían provenir del cacique y su pueblo), y (8) veinte huevos y tres cargas de pescado cada viernes de la Cuaresma (también provenientes del cacique y su comunidad). Ver AGI, Patronato, 196, r. 8, fols. lv-2r (29 agosto de 1574).

${ }^{67}$ AGN, Visitas Boyacá, 29, Ni, fol. 8v. A los indígenas de pueblos sin iglesias usualmente se les ordenaba que fueran a misa en la iglesia más cercana. Por ejemplo, a los indígenas de Foacá se les exigía que caminaran dos kilómetros hasta la iglesia de Samacá. Aunque esta distancia no era excesiva, es difícil imaginar como esa iglesia podía albergar a todos los indígenas de ambos pueblos. Al mismo tiempo, había ochenta y tres indígenas tributarios en Foacá y más de cien en Samacá.

${ }^{68}$ Germán Colmenares, La Provincia de Punja en el Nuevo Reino de Granada (Bogotá: Universidad de los Andes, 1970), 13.

${ }^{69}$ AGN, Visitas Boyacá, 17, fol. 90r. Claro que esta situación no era única. En otros lugares del Nuevo Mundo, incluido Méjico, había frailes encargados de un gran número de personas. El virrey de Méjico, Martín Enríquez, consideraba que era razonable que un religioso sirviese a mil indígenas. Ver Schwaller, Church and Clergy, 91.
} 


\section{El improbable remedio}

Los múltiples obstáculos a la conversión de indígenas son reconocidos claramente por el más casual de los observadores de la Nueva Granada del siglo XVI, y los documentos coloniales revelan un amplio rango de posibles soluciones que provenían de varias fuentes, desde el presidente y los oidores de la Audiencia hasta sacerdotes seglares, encomenderos, miembros de varias órdenes religiosas y arzobispos. Quizás la más meditada lista de propuestas provino de la correspondencia de 1608 del jesuita Martín de Funes, quien sugería una serie de remedios que llevarían a cambios profundos en las vidas espirituales de los nativos de la Nueva Granada ${ }^{70}$. Tales cambios, insistía, se necesitaban de manera urgente, ya que el cristianismo no tenía un control firme de la población indígena en ningún lugar de las Indias, ni siquiera en Méjico. Funes reconocía que muchos de los indígenas del territorio muisca habían tenido algún contacto con la "fe verdadera", y muchos parecían aceptar al cristianismo con gran entusiasmo. Sin embargo, también advertía que el entusiasmo que los nativos mostraban ante las celebraciones cristianas y las ceremonias públicas como el bautismo no debían confundirse con una conversión genuina. Esto se confirmó veinte años después en el informe de un grupo de jesuítas de Tunja quienes se sorprendieron al descubrir que, aunque habían sido bautizados, los indígenas no comprendían lo que dicha ceremonia significaba ${ }^{71}$.

Para remediar la situación, Funes propuso que se dieran varias reformas; como es de esperarse, insistió en que fuesen los jesuítas quienes las llevasen a cabo ${ }^{72}$. También pidió la consolidación de un programa masivo para cambiar la localización de las poblaciones muiscas, un plan que se había discutido desde 1540 pero que sólo se había puesto en marcha a gran escala recientemente ${ }^{73}$. Ocho años antes que Funes

\footnotetext{
${ }^{70}$ ARSI, Congregationes Provinciarum, 52, fols. 197r-197v.

${ }^{71}$ Pacheco, La Evangelización, 497.

${ }^{72}$ Funes citó la escasez de clérigos y los pésimos ejemplos que ellos daban como los dos obstáculos primordiales para la conversión. Culpaba tanto a los sacerdotes seglares como a los regulares por su falta de fe, devoción y diligencia. Para hallar un remedio, Funes sugería que los jesuitas fuesen llamados para educar a todo el clero. Pidió más religiosos para la región, y propuso que los jesuitas se encargasen de la educación de la élite indígena. También pidió que se establecieran colegios donde los indígenas pudieran ser educados.

${ }^{73}$ En 1549 la Corona ordenó que los oficiales de la Nueva Granada examinaran la posibilidad de localizar a la población indígena en determinados pueblos para facilitar su conversión al cristianismo. Ver Juan Villamarín y Judith Villamarín, "Chibcha Settlement Under Spanish Rule: 1537-1810," en Social Fabric and Spacial Structure in Colonial Latin America, ed. David J. Robinson (Syracuse: University Microfilms International, 1979 ), 25.
} 


\section{FRONTERAS}

hubiera esbozado su propuesta, el visitador Luis Enríquez fue encargado de reubicar a la población muisca. Los registros de la inspección revelan que Enríquez ordenó la reducción de un total de 154 pueblos de la provincia a 55 resguardos ${ }^{74}$.

Si bien es posible que la congregación forzosa de las poblaciones muiscas fuera la solución más apropiada para una verdadera conversión de la población indígena, los resultados no se sintieron inmediatamente. De hecho, el programa de reubicación a menudo fue opacado por intentos más agresivos y menos efectivos de liberar a la región de la idolatría. Referiré a continuación algunas de las campañas que se dieron para erradicar la idolatría indígena.

Los conquistadores de la Nueva Granada y sus primeros colonos no tardaron en notar que en los santuarios funerarios muiscas había gran cantidad de oro y de piedras preciosas ${ }^{75}$. La mayor parte del botín que los primeros conquistadores saqueaban consistía en oro, mantas de algodón y esmeraldas extraídas de dichos lugares, una práctica que continuó a lo largo de la primera década de la conquista española $^{76}$. Sin embargo, luego del establecimiento de la Audiencia en 1550, los oficiales españoles intentaron restringir tales prácticas. Una provisión real de 1551 prohibía el saqueo de cementerios nativos. Infortunadamente, la tentación de las riquezas ocultas y el reconocimiento de que los intentos por prevenir tales actividades habían fallado, eventualmente alentó a los oficiales de la audiencia de Santa Fe a permitir que ciertos individuos desenterraran tumbas muiscas. En un principio se prohibió a los indígenas que participaran en tal actividad; con el tiempo se decidió que aquellos que ofreciesen sus servicios "voluntariamente" podían ayudar, si eran provistos con raciones alimenticias adecuadas y se les pagaba un pequeño salario ${ }^{77}$.

\footnotetext{
${ }^{74}$ El programa tuvo un éxito limitado. Para un análisis detallado del sistema de los resguardos, ver Francis. "The Muisca Indians," 199-206.

${ }^{75}$ El cronista del siglo XVI, Pedro de Aguado, hace referencia a este hecho en su recuento de la búsqueda de Hernán Pérez de Quesada en 1541 de la Casa del Sol, un lugar donde los señores muiscas eran enterrados, y a donde los nativos viajaban desde muy lejos para adorar a sus ancestros. Ver Aguado, Recopilación Historial, vol. l,lib. 3, cap. 14,317.

${ }^{76}$ En particular, los conquistadores descubrieron riquezas increíbles en el famosoTemplo Muisca de Sogamoso. Para más detalles ver Fray Pedro Simón, Noticias Historiales: De las Conquistas de Tierra Firme en las Indias Occidentales (Bogotá: Banco Popular, 1982), 3: 260, 261.

${ }^{77}$ Triana, Las Lenguas Indígenas, 218. Los indígenas que prestaban sus servicios voluntariamente recibían un tomín diario.
} 
El saqueo de cementerios muiscas fue común durante el primer periodo de la conquista y para mayo de 1569, tales prácticas eran permitidas por la Audiencia. Como reacción a lo que él describía como una "terrible ofensa a nuestro Dios", el visitador Juan López de Cepeda sancionó la apropiación de oro, piedras preciosas y mantas que los indígenas dieran como ofrendas a sus ídolos ${ }^{78}$. López concedió un periodo de gracia de quince días para que los indígenas renunciasen a sus ídolos y abandonasen toda práctica pagana; sin embargo, afirmó que al terminar el periodo de gracia, la expropiación de todas las diabólicas ofrendas sería legal ${ }^{79}$. A cambio de esto, quienes confiscaban las ofrendas debían pagar una quinta parte de los bienes como impuesto. Se alentó a los miembros del clero a que confiscasen las dádivas ilícitas, especialmente aquellas de oro ${ }^{80}$. Como es lógico, hubo muchos abusos. A menudo los indígenas eran torturados y forzados a entregar su oro y sus mantas, y a revelar la ubicación secreta de sus santuarios ${ }^{81}$. Por ejemplo, parece que el sacerdote de Lenguasaque ofreció favorecer el escape de un indígena, acusado de envenenar a una mujer española, si él le revelaba la ubicación de su santuario ${ }^{82}$. Es más, sólo un pequeño porcentaje de la riqueza extraída de los cementerios muiscas fue declarada. Para 1580, se estimaba que el robo ilegal de santuarios nativos le costó a la Corona más de 200.000 pesos en impuestos que nunca fueron pagados. Casi cualquiera perpetraba tales robos: los oficiales de la Audiencia, el arzobispo, miembros del clero, incluso el tesorero real Juan Antonio de Vilches ${ }^{83}$.

En 1577 la Audiencia de Santa Fe lanzó una breve pero violenta cruzada contra la idolatría en el territorio muisca. Aunque la campaña fue breve, resultó notablemente rentable para las arcas de la Audiencia. En sólo 10 pueblos de las jurisdicciones de Santa Fe y Tunja, se confiscaron cerca de 44.129 pesos de los santuarios muiscas, un estimado moderado teniendo en cuenta que muchas de las ganancias nunca se

\footnotetext{
${ }^{78}$ Colmenares, La Provincia de Tunja, 25. Cinco años después, en 1574, un decreto real ordenaba que todas las ofrendas a los ídolos debían ser confiscadas y quemadas. Ver AGI, Patronato, 196, r. 8, fol. 6r.

${ }^{79}$ AGN, Caciques e Indios, n. 28, fol. 614r.

${ }^{80}$ AGI, Patronato, 27, r. 34, carta del fiscal Francisco Guillen Chaparro, fol. lv.

${ }^{81}$ Esperanza Gálvez Piñal, La Visita de Monzón y Prieto de Orellana al Nuevo Reino de Granada (Sevilla: Escuela de Estudios Hispanoamericanos, 1974), 103-4.

${ }^{82}$ AGN, Caciques e Indios, 24, n. 1, fol. 20r. Para ver más detalles de este caso, ver Francis, "The Muisca Indians," 88-93.

${ }^{83}$ Gálvez Piñal, La Visita de Monzón, 13-74.
} 


\section{FRONTERAS}

declaraban oficialmente ${ }^{84}$. Cinco años después, en 1582, una investigación sobre los abusos cometidos durante la campaña reveló que muchos indígenas habían sido torturados durante extensos interrogatorios. Se sugirió que una víctima, el cacique de Duitama, se suicidó debido al tormento físico que había sufrido a manos de oficiales españoles, los cuales buscaban desesperadamente la ubicación de los santuarios de Duitama $^{85}$.

Menos de veinte años después, en 1595, el oidor Egas de Guzmán lidera otra campaña para el exterminio del paganismo. Sin embargo, como en las persecuciones de 1577, esta inspección se interesó más en localizar las riquezas escondidas de los santuarios muiscas que en convertirse en una genuina erradicación de las prácticas de idolatría. Claramente ninguna de las campañas se centraba en la vida espiritual de los indígenas ${ }^{86}$.

En general, los intentos para erradicar la idolatría tuvieron poco éxito. De hecho probablemente alentaron una cierta aversión por el cristianismo, y al mismo tiempo hicieron que las prácticas religiosas muiscas se realizaran secreta y subterráneamente. No se ha determinado si la violencia y la coerción de estas campañas en la Nueva Granada generaron el resentimiento, la ira y la desconfianza características de las campañas contra las religiones nativas del Perú ${ }^{87}$. Es claro que si las campañas

\footnotetext{
${ }^{84}$ Colmenares, La Provincia de Tunja, 25; Idem, Historia Económica y Social de Colombia, 1537-1719, 2a. ed. ( Medellín: La Carreta, 1975), 60. Para el mejor recuento de la campaña de 1577, ver Vicenta Cortés, "Visita a los Santuarios Indígenas de Boyacá," Revista Colombiana de Antropología 9 (1960), 199-273.

${ }^{85}$ Colmenares, Historia Económica y Social, 60. Los rumores del carácter viciado de la campaña crecieron rápidamente por las provincias de Santa Fe y Tunja. Cuando Diego Hidalgo de Montemayor, el factor de la campaña de 1577, llegó a Tunja para empezar sus interrogatorios, ya se sabía que la campaña había arribado a los pueblos vecinos, y muchos de los caciques e indios principales habían huido de la región. Ver AGN, Real Hacienda, 21, fol. 740r. Hidalgo ordenó que los encomenderos de Tunja encontrasen a sus caciques en un periodo de dos días; aquellos que fallaran tendrían que pagar una multa de cuatro días de salario (probablemente el del factor).

${ }^{86}$ Los detalles de la visita de Guzmán se pueden encontrar en Francis, "The Muisca Indians", 258-74. Un recuento detallado de la inspección de Guzmán al pueblo de Iguaque se puede ver en J. Michael Francis," 'In the Service of God, I Order These Temples of Idolatrous Workship Razed to the Ground': Extirpation of Idolatry and the Search for the Santuario Grande of Iguaque," en Colonial Lives: Documents on Latin American History, 1550-1850, ed. Richard Boyer y Geoffrey Spurling (Oxford: Oxford University Press, 2000), 39-53.

${ }^{87}$ Ver Kenneth Mills, Idolatry and Its Enemies: Colonial Andean Religion and Extirpation, 1640-1750 (Princeton: Princeton University Press, 1997).
} 
buscaban erradicar genuinamente la idolatría, fallaron. Cuando Juan de Valcárcel llevó a cabo su inspección general en la provincia de Tunja, se informó que las prácticas paganas se habían extendido y aún se necesitaba mucho trabajo para convertir a la menguada población nativa a la cristiandad.

\section{Conclusión}

A pesar de la aparente persistencia de las prácticas religiosas muiscas, la complejidad de los cambios religiosos en la Nueva Granada no debe ser menospreciada. Es poco lo que se sabe respecto a la respuesta del pueblo muisca a la nueva fe, a cómo cambiaron sus creencias y cómo evolucionó el cristianismo en el periodo colonial. Centrarse demasiado en las fallas de los sacerdotes y frailes de la Nueva Granada implica olvidar los testimonios de cientos de nativos que insistían en que eran cristianos, a los hombres y mujeres que caminaban largas distancias para ir a misa, y a las múltiples confraternidades indígenas que surgieron a mediados del siglo XVII. Hay muchos ejemplos de pueblos muiscas que lucharon para proteger a sus sacerdotes o que solicitaron a la Audiencia y al arzobispo la presencia permanente de un religioso en su población. También hay otros que se quejaban del estado lamentable de sus iglesias locales. Todos estos elementos deberían evitar que los historiadores reduzcan la historia religiosa de la Nueva Granada a un simple catálogo de triunfos y derrotas. Sin embargo, parece improbable que, como afirmó recientemente María Imelda López Avila, la religión muisca desapareciera del todo en el siglo XVI a causa de la cuidadosa y cercana supervisión de los clérigos españoles $^{88}$. Claramente ese no fue el caso.

El hecho que la idolatría indígena persistiese durante todo el primer periodo de la colonia no resulta sorprendente, especialmente al considerar la naturaleza precaria de la vida colonial, la constante amenaza de las enfermedades, las pérdidas de tierras, la forzada reubicación de ciertas comunidades y el peso de las exigencias tributarias, la mita y los servicios personales ${ }^{89}$. Además, el cristianismo no fue

\footnotetext{
${ }^{88}$ María Imelda López Avila, "Expresiones de la Vida Espiritual y Colonial de los Muiscas en el Siglo XVI," en Historia y Cultura Populares, ed. Pablo Mora Calderón y Amado Guerrero Rincón (Tunja: Centro de Investigación de Cultura Popular del ICBA, 1989).

${ }^{89}$ Nancy Farriss, Maya Society Under Colonial Rule: The Collective Enterprise of Survival (Princeton: Princeton University Press, 1984), 256.
} 
accesible a la vasta mayoría de los habitantes de Tunja. La ausencia de iglesias en muchos de los pueblos y la escasez de clérigos en la región probablemente implicaban que el Dios cristiano se encontraba muy lejos de la vida cotidiana de los indígenas. Como tal, resulta lógico que las prácticas precolombinas sobrevivieran. Basta referirse al informe de Juan de Valcárcel luego de su inspección de 1636 a la provincia de Tunja para encontrar evidencias abrumadoras de la persistencia de los rituales muiscas ${ }^{90}$. Sería poco realista esperar que en cien años un número reducido de frailes, muchos de los cuales no tenían una preparación adecuada y desconocían total o parcialmente las lenguas nativas, pudiese supervisar e instruir a una población tan numerosa y dispersa. Esto no implica, desde luego, que las creencias muiscas permanecieran intactas a lo largo del primer siglo de la colonia, ni sugiere que los muiscas siempre se opusieron a la imposición de una nueva fe. Es probable que estudios futuros muestren que los muiscas aceptaron ciertos aspectos del cristianismo en determinados momentos, y que ellos cumplieron un papel activo e importante en la consolidación de la religión colonial en la Cordillera Oriental de Colombia. Quizás la lección más significativa que los futuros estudios van a revelar sea que la transformación de la sociedad colonial fue un proceso gradual y muy complejo; y si bien Orlando Fals Borda tenía razón al afirmar que la Iglesia Católica fue 1a institución más importante e influyente para la vida diaria de la población de Tunja. cien años después de la conquista el proceso estaba lejos de concluir ${ }^{91}$.

\footnotetext{
${ }^{90}$ Rojas, 21-25.

${ }^{91}$ Fals Borda, quien llevó a cabo extensos trabajos de campo por toda la Cordillera Oriental de Colombia en la década de 1950, concluyó que la devoción al catolicismo era tan fuerte que "la homogeneidad religiosa [era] uno de los principales y más influyentes rasgos de Boyacá". Ver Orlando Fals Borda, El Hombre y la Tierra en Boyacá, 2a. ed. (Bogotá: Punta de Lanza, 1973), 31.
} 